OPEN ACCESS

Edited by:

Juarez Antonio Simões Quaresma,

Federal University of Pará, Brazil

Reviewed by:

Tianlei Ying,

Fudan University, China

Qibin Leng,

Institut Pasteur of Shanghai (CAS),

China

Takaaki Koma,

Tokushima University Graduate

School of Medical Sciences, Japan

*Correspondence:

Thomas W. Geisbert

twgeisbe@utmb.edu;

Ilhem Messaoudi

imessaou@uci.edu

tThese authors have contributed equally to this work.

Specialty section: This article was submitted to Microbial Immunology, a section of the journal

Frontiers in Immunology

Received: 19 August 2017 Accepted: 05 October 2017 Published: 26 October 2017

Citation:

Menicucci AR, Versteeg $K$, Woolsey C, Mire CE, Geisbert JB, Cross RW, Agans KN, Jankeel A, Geisbert TW and Messaoudi I (2017) Transcriptome Analysis of Circulating Immune Cell Subsets Highlight the Role of Monocytes in Zaire Ebola Virus Makona Pathogenesis.

Front. Immunol. 8:1372. doi: 10.3389/fimmu.2017.01372

\section{Transcriptome Analysis of Circulating Immune Cell Subsets Highlight the Role of Monocytes in Zaire Ebola Virus Makona Pathogenesis}

\author{
Andrea R. Menicucci ${ }^{1 \dagger}$, Krista Versteeg ${ }^{2,3+}$, Courtney Woolsey ${ }^{2,3}$, Chad E. Mire ${ }^{2,3}$, \\ Joan B. Geisbert ${ }^{2,3}$, Robert W. Cross ${ }^{2,3}, K_{\text {Krystle N. Agans }}^{2,3}$, Allen Jankeel ${ }^{4}$, \\ Thomas W. Geisbert ${ }^{2,3 *}$ and Ilhem Messaoudi ${ }^{4 *}$ \\ ${ }^{1}$ Division of Biomedical Sciences, University of California, Riverside, Riverside, CA, United States, ${ }^{2}$ Galveston National \\ Laboratory, Galveston, TX, United States, ${ }^{3}$ Department of Microbiology and Immunology, University of Texas Medical \\ Branch, Galveston, TX, United States, ${ }^{4}$ Department of Molecular Biology and Biochemistry, College of Biological Sciences, \\ University of California, Irvine, Irvine, CA, United States
}

Existing models of Ebola virus disease (EVD) suggest antigen-presenting cells are initial targets of Zaire ebolavirus (ZEBOV). In vitro studies have shown that ZEBOV infection of monocytes and macrophages results in the production of inflammatory mediators, which may cause lymphocyte apoptosis. However, these findings have not been corroborated by in vivo studies. In this study, we report the first longitudinal analysis of transcriptional changes in purified monocytes, T-cells, and B-cells isolated from cynomolgus macaques following infection with ZEBOV-Makona. Our data reveal monocytes as one of the major immune cell subsets that supports ZEBOV replication in vivo. In addition, we report a marked increase in the transcription of genes involved in inflammation, coagulation, and vascular disease within monocytes, suggesting that monocytes contribute to EVD manifestations. Further, genes important for antigen presentation and regulation of immunity were downregulated, potentially subverting development of adaptive immunity. In contrast, lymphocytes, which do not support ZEBOV replication, showed transcriptional changes limited to a small number of interferon-stimulated genes (ISGs) and a failure to upregulate genes associated with an antiviral effector immune response. Collectively, these data suggest that ZEBOVinfected monocytes play a significant role in ZEBOV-Makona pathogenesis and strategies to suppress virus replication or modify innate responses to infection in these cells should be a priority for therapeutic intervention.

Keywords: Ebola, hemorrhagic fever, pathogenesis, RNASeq, monocytes

\section{INTRODUCTION}

Ebolaviruses (EBOV) are among the deadliest re-emerging viruses; causing hemorrhagic fever with case fatality rates (CFRs) ranging from 40 to $90 \%$ depending on the species. Ebola virus disease (EVD) is characterized by an excessive inflammatory response, lymphocyte apoptosis, vascular impairment, hemorrhage, and coagulation defects leading to multi-organ failure and shock $(1,2)$. Zaire ebolavirus (ZEBOV) is responsible for the highest CFRs and most outbreaks, 
including the largest epidemic that originated December 2013 in West Africa. The ZEBOV variant responsible for this epidemic, Makona, affected over 28,600 individuals and claimed over 11,300 lives (3).

Despite its impact on human health, the molecular basis for EVD is incompletely understood. Much of what is known about ZEBOV pathogenesis has been acquired through infectious studies in nonhuman primates (NHPs), particularly cynomolgus and rhesus macaques. Studies in macaques have shown that monocytes, macrophages, and dendritic cells (DCs) are the initial targets of ZEBOV (4). Our understanding of how ZEBOV impacts function and behavior of DC and monocytes/macrophages has been primarily extrapolated from in vitro infections. Data from these studies show that in vitro infection of monocytes and macrophages with ZEBOV triggers a robust expression of inflammatory mediators including IL- $1 \beta$, IL-6, IL-8, MIP- $1 \alpha$, MIP-1 $\beta$, MCP-1, and TNF $\alpha$ (5-7), several of which have been detected in the plasma of humans and animal models following ZEBOV infection $(5,8-12)$. Inflammatory mediators released by monocytes may also contribute to the impairment of the vascular system and disseminated intravascular coagulation as well as lymphocyte death (13-16). However, whether monocytes are the major contributors to inflammation following ZEBOV infection in vivo remains to be elucidated. Moreover, the susceptibility of monocytes to ZEBOV remains contradictory. Some in vitro studies reported successful ZEBOV replication in both primary monocytes and macrophages $(6,7,15)$; others indicated ZEBOV entry is delayed in primary compared to differentiated monocytes and THP-1 cells are refractory to entry until PMA-induced differentiation (17).

Severe lymphopenia is a hallmark of ZEBOV infection $(4,12$, $18,19)$, observed as a loss of peripheral blood $\mathrm{CD}^{+}$and $\mathrm{CD}^{+}$ $\mathrm{T}$-cells, as well as natural killer cells in cynomolgous macaques $(20,21)$ and humans $(22)$. The loss of B-cells has been controversial with some NHP studies reporting apoptosis of B lymphocytes (15), while others observe no changes in B-cell counts (20). In vivo and in vitro studies using TUNEL staining and transmission electron microscopy confirm apoptosis as the main mechanism of lymphocyte loss during ZEBOV infection $(14,15)$. Furthermore, while analysis of ZEBOV-infected NHP tissues shows the presence of ZEBOV antigens within phagocytic cells 3-4 days post challenge, no ZEBOV antigens have been observed in T- and B-cells throughout infection. This suggests lymphocyte apoptosis during $\mathrm{ZEBOV}$ infection is not due to direct viral replication but rather inflammatory mediators, such as TNF $\alpha$, nitric oxide, and reactive oxygen species. Presumably these cytokines and chemokines are produced in response to infection of phagocytes and other cells as well as immunosuppressive EBOV peptides (15, 23-25). However, the effects of ZEBOV infection and these mediators on $\mathrm{T}$ - and B-cell function remains incompletely defined.

Although many genomic studies have provided us with insight into the global transcriptional changes as disease progresses, studies that elucidate the role of individual immune cell subsets in viral pathogenesis are lacking. In this study, we used RNA sequencing (RNA-Seq) to uncover longitudinal gene expression profiles within monocytes, T-cells, and B-cells purified from ZEBOV-Makona infected cynomolgus macaque peripheral blood mononuclear cells (PBMC) at different times post infection. Our data identify monocytes as one of the major targets of infection in vivo. Moreover, gene expression profiles at later stages of disease strongly support the role of monocytes as a significant source of inflammatory cytokines and chemokines during ZEBOV infection. In contrast, lymphocytes exhibited limited transcriptional activity and lacked significant signs of an effective adaptive immune response.

\section{MATERIALS AND METHODS}

\section{Study Design}

This study was designed to elucidate the role of specific immune cell subsets in mediating ZEBOV-Makona pathogenesis. For this purpose, monocytes, T-cells, and B-cells were isolated by magnetic bead separation from PBMC collected on days $0(n=10)$, $1(n=5), 2(n=4), 3(n=3), 4(n=2), 5(n=2)$, and $6(n=4)$ post ZEBOV-Makona infection from cynomolgus macaques (26). Challenges with allowable volumes of blood collection and cell separation within a Biosafety Level 4 (BSL-4) lab precluded us from having $n>2$ for days 4 and 5 post infection. RNA-Seq was used to measure host gene expression changes within each cell subset.

\section{Virus and Challenge}

A laboratory seed stock of the Makona variant isolate H.sapiens-tc/GIN/2014/Makona-Gueckedou-C07 (accession number KJ660347.2) was grown from the serum of a 2014 fatal human case in Guékédou, Guinea and passaged twice in authenticated Vero E6 cells (ATCC, CRL-1586). This clone was selected because it is one of the earliest and well characterized isolates from the EBOV epidemic in West Africa that was also used in a recent NHP study (27). Ten healthy, filovirus-negative male cynomolgus macaques (Macaca fascicularis) 3-5 years of age and between 4 and $8 \mathrm{~kg}$ were challenged with 1,000 pfu of ZEBOV-Makona intramuscularly with the dose divided equally into the left and right quadriceps. Animals were housed in the BSL-4 laboratory in the Galveston National Laboratory and monitored post challenge for clinical signs of disease including temperature, weight loss, behavioral changes, changes in blood count, and blood chemistries.

\section{Sample Collection and PBMC Isolation}

Blood was collected by venipuncture into EDTA tubes. To isolate PBMC, whole blood was centrifuged over Histopaque (Sigma-Aldrich, St. Louis, MO, USA) using AccuSpin Tubes (Sigma-Aldrich, St. Louis, MO, USA) at $\sim 400 \times g$ for $45 \mathrm{~min}$, room temperature with no brake. PBMC were counted on a TC20 Automated Cell Counter (Bio-Rad, Hercules, CA, USA).

\section{Magnetic Bead Cell Separation}

Peripheral blood mononuclear cells underwent sequential separations using magnetic microbeads (Miltenyi Biotec, San Diego, CA, USA) as described in Figure S1 in Supplementary Material. PBMC were initially stained with anti-CD2 microbeads to isolate $\mathrm{T}$ and NK cells. The CD2 negative cell population was then 
stained with anti-CD20 microbeads to isolate the B-cell population. The CD20 negative fraction was collected and stained with CD14 microbeads to isolate monocytes. Purity of the fractions was confirmed using flow cytometry (Table S1 in Supplementary Material). All samples were acquired using a BD FACS Canto-II (Becton Dickinson Biosciences, San Jose, CA, USA) using BD FACS Diva software. Live cells were identified by FSC and SSC and a minimum of 50,000 events were collected for each sample. Data was analyzed using FlowJo Analysis Software (FlowJo LLC, Ashland, OR, USA) and Prism Software (GraphPad Software, Irvine, CA, USA). Only samples with purity above the cutoff of $60 \%$ were included in the analysis. The number of samples available at each time point were as follows: monocyte samples on days $0(n=5), 1(n=4), 2(n=4), 3(n=2), 4(n=2)$, and $6(n=3)$ post infection; T-cell samples on days $0(n=7), 1(n=4), 2(n=4)$, $3(n=2), 4(n=2), 5(n=2)$, and $6(n=3)$ post infection; and B-cell samples on days $0(n=2), 1(n=4), 2(n=4)$, and $6(n=4)$ post infection. Unfortunately, a sufficiently pure DC population could not be obtained; therefore, in this study, we focused on monocytes, T-cells, and B-cells.

\section{Library Preparation for RNA-Seq}

RNA from each cell subset was isolated using Zymo Research Direct-zol RNA mini-prep (Zymo Research, Irvine, CA, USA) per manufacturer's instructions. RNA concentration and integrity was determined using an Agilent 2100 Bioanalyzer (Agilent Technologies, Santa Clara, CA, USA). Ribosomal RNA (rRNA) was depleted using the ClontechRibo-Gone rRNA Removal kit. Libraries were constructed using the ClontechSMARTer Stranded RNA-Seq kit (Takara Bio Inc., Kusatsu, Shiga, Japan). First, rRNA-depleted RNA was fragmented and converted to double stranded cDNA. Adapters were ligated and the $\sim 300$ base pair long fragments were then amplified by PCR and selected by size exclusion. Each library was prepared with a unique indexed primer for multiplexing. In order to ensure proper sizing, quantitation, and quality prior to sequencing, libraries were analyzed on the Agilent 2100 Bioanalyzer. Multiplexed libraries were subjected to single-end 100 base pair sequencing using the Illumina HiSeq2500 platform (Illumina, San Diego, CA, USA).

\section{Bioinformatic and Statistical Analysis}

Data analysis was performed with the RNA-Seq workflow module of the systemPipeR package available on Bioconductor (28). RNA-Seq reads were demultiplexed, quality filtered, and trimmed using Trim Galore with an average phred score cutoff of 30 and minimum length of 75bp. Three base pairs from the $5^{\prime}$ end were trimmed as per Clontech's instruction. Quality reports were generated with the FastQC function. Because the complete genome annotation for $M$. fascicularis is not available, the Macaca mulatta genome sequence (Macaca_mulatta.MMUL_1.dna.toplevel.fa) and annotation file from Ensembl (Macaca_mulatta.MMUL_1.78.gtf) was used. In order to determine the level of viral transcription at different time points, the ZEBOV variant Makona genome (H.sapiens-wt/GIN/2014/Makona-Gueckedou-C07) from Virus Pathogen Resource was adjoined to the M. mulatta reference. ZEBOV open reading frames (ORFs), intergenic regions (IGRs), and leader and trailing sequences were defined based on the
ZEBOV-Makona genome annotation GTF file: NP (470-2689), VP35 (3129-4151), VP40 (4479-5459), GP (6039-8068), VP30 (8509-9375), VP24 (10345-11100), L (11581-18219), Leader (1-469), IGR_NP_VP35 (2690-3128), IGR_VP35_VP40 (4152-4478), IGR_VP40_GP (5460-6038), IGR_GP_VP30 (8069-8508), IGR_VP30_VP24 (9376-10344), IGR_VP24_L (11101-11580), and Trailing (18220-18959). RNA-Seq reads were mapped with the alignment suite Bowtie2/Tophat 2 against a reference genome containing both $M$. mulatta and ZEBOVMakona genome sequences. Raw expression values in the form of gene-level read counts were generated with the summarizeOverlaps function, counting only the reads overlapping exonic regions of genes, and discarding reads mapping to ambiguous regions of exons from overlapping genes. Normalization and statistical analysis of differentially expressed genes (DEGs) was performed using the edgeR package, which normalizes reads by the trimmed mean of $M$ values method. RNA-seq data presented in this article were submitted to the National Center for Biotechnology Information Sequence Read Archive (BioProject accession PRJNA412109). DEGs were defined as those with a fold change $\geq 2$ and a false discovery rate (FDR) corrected $p$-value $\leq 0.05$ compared to 0 days post-infection (DPI). Only protein coding genes with human homologs and an average 5 reads per kilobase of transcript per million mapped reads (RPKM) were included for further analysis. Reads mapping to the ZEBOV-Makona genome were normalized as RPKM. Heatmaps, venn diagrams, and a violin plot were generated using $\mathrm{R}$ packages gplot, DESeq2, VennDiagram, and ggplot2. Heatmaps for gene expression represent the absolute normalized expression (RPKM); range of colors is based on scaled and centered RPKM values of the entire set of genes (red represents increased expression while blue represents decreased expression). Heatmaps for functional enrichment of DEGs represent $-\log _{10}$ (FDR-corrected p-value); range of colors is based on the lowest and highest $-\log _{10}$ (FDR) values for the entire set of terms; the number of DEGs mapping to each functional enrichment term each day is listed within each box; blank boxes represent no statistical significance.

Differentially expressed genes detected in monocytes 6 DPI were compared to the transcriptional profile of monocytederived macrophages (MDMs) and macrophages differentiated from human induced pluripotent stem cells following 6-h lipopolysaccharide (LPS) stimulation (29) using R package VennDiagram.

\section{Functional Enrichment}

Functional enrichment of DEGs was done to identify clusters of genes mapping to specific biological pathways, specifically Gene Ontology (GO) terms and Diseases (by Biomarkers) using MetaCore $^{\mathrm{TM}}$ (Thomson Reuters, New York, NY, USA). Significant functional enrichment terms were defined as those with a FDR corrected $p$-value $\leq 0.05$. Since this software requires human gene identifiers for analysis, rhesus DEGs were mapped to human homologs using BioMart.

\section{Statistical Analysis}

Statistical analysis of viral genome copy number; hematology and clinical chemistry data; cytokine, chemokine, and growth 
factor data; and flow cytometry data were carried out using the SAS software, PROC MIXED (Figure S2 in Supplementary Material; Table 1). A repeated measures analysis was used to model each of the dependent variables. Intra-animal correlation was modeled using a compound symmetric variance-covariance structure. In some cases, a linear trend was used for the mean. In other cases, in which a linear trend was not a good fit, a nonparametric trend was used where each time point was modeled by its own mean. Missing data were handled by using maximum likelihood algorithms to fit the model. When a linear model was an adequate trend, the $p$-value for the estimated slope was reported. When each time point was modeled by its own mean, the mean response at each non-zero time point was contrasted with the mean response at the zero time point. Holm's multiple comparison method was used to adjust the $p$-values for each contrast (30).

\section{RESULTS}

\section{Disease Course Following Makona Infection}

Viremia was first detected 3 DPI and increased as disease progressed (Table 1). Clinical signs (fever, rash, bleeding), liver and kidney dysfunction, decreased platelet count, and increased inflammatory cytokine and chemokine levels were evident 5-6 DPI (Table 1; Figure S2A in Supplementary Material). Although the number of circulating monocytes, DCs, and NK cells remained relatively stable (Figure S2B in Supplementary Material), we observed a transient increase in the number of activated monocytes 4 DPI (Figure S2B in Supplementary Material). In contrast, the number of $\mathrm{CD}^{+} \mathrm{T}$-cells, $\mathrm{CD}^{+} \mathrm{T}$-cells, and $\mathrm{B}$-cells as well as their naïve and memory subsets significantly decreased as infection progressed (Figure S2B in Supplementary Material). Transcriptional analysis of PBMC following infection shows the number of DEGs correlated with viral loads, with the largest changes detected 6 DPI (Figure 1A). Functional enrichment revealed that DEGs upregulated 6 DPI play a role in innate immunity, inflammation, and cell death, while downregulated DEGs are important for cell motility, signaling, and adaptive immunity (Table 2). These findings are described in greater detail in our earlier study (26).

\section{Monocytes Support ZEBOV-Makona Replication and Are Major Contributors of Inflammation}

To investigate the impact of ZEBOV-Makona infection within monocytes, we determined longitudinal changes in gene expression profiles using RNA-Seq. The average purity of monocytes analyzed at the various time points was $>70 \%$ (Table $S 1$ in Supplementary Material). Transcriptional changes correlated with ZEBOV reads (Figure 1A). We further investigated the total number of viral reads mapping to each ZEBOV ORF and IGR within purified monocytes (Figure 1B). At 6 DPI, viral transcripts were abundant with ORF $\mathrm{L}$ and the trailing end region having the lowest average RPKM values, while ORFs GP and NP had the highest. Based on Euclidean distances, the viral transcript profile in monocytes was most comparable to that observed in PBMC (Figure S3 in Supplementary Material).

A small number of DEGs was detected 3 DPI within monocytes, which slightly increased 4 DPI, and then considerably increased 6 DPI (Figure 1A). DEGs detected 3 and 4 DPI enriched to GO terms associated with innate immunity such as type I IFN signaling pathways (Figures 2A,B). Only 2 DEGs were downregulated 3 DPI: $H B B$ (Hemoglobin Subunit Beta) and transcription factor JUN (Jun Proto-Oncogene), which play an important role in inflammation (31) (Figure 2C). The 6 DEGs upregulated only 4 DPI included: FAM 3 C (Family With Sequence Similarity 3 Member C), which encodes Interleukin-Like EMT inducer; HIP1R (Huntingtin Interacting Protein 1 Related), a component of clathrin-coated vesicles; signaling adaptor BANK1 (B-Cell Scaffold Protein with Ankyrin Repeats); and receptor FCRL1 (FC Receptor -Like 1) (Figure 2C). Although these genes are mostly known to be expressed in B-cells, they are also expressed in activated monocytes and macrophages (32-36). Their increased expression correlated with increased frequency of CD16 + nonclassical monocytes 4 DPI (Figure S2B in Supplementary Material).

Most of the 22 DEGs that were upregulated throughout infection in monocytes (Figure 2A) were interferon-stimulated genes (ISGs) important in anti-viral defense (37), notably GBP1 (Guanylate Binding Protein 1), OAS2 (2'-5'-Oligoadenylate Synthetase 2), MX1 (MX Dynamin-Like GTPase 1), and IFIT1-3 (Interferon-Induced Protein with Tetratricopeptide Repeats

TABLE 1 | Summary of average clinical data and flow cytometry data during ZEBOV-Makona infection.

\begin{tabular}{|c|c|c|c|c|c|c|}
\hline Days post infection & 1 & 2 & 3 & 4 & 5 & 6 \\
\hline Viral load (genome copy/mL) & 50 & 50 & 1.89E06 & $3.12 \mathrm{E} 08$ & 1.37E09 & 3.68E10 \\
\hline Rash (fraction of animals) & & & & & $2 / 4$ & $3 / 4$ \\
\hline Anorexia, depression (fraction of animals) & & & & & & $4 / 4$ \\
\hline Weakness (fraction of animals) & & & & & & $2 / 4$ \\
\hline Hemorrhage (fraction of animals) & & & & & & $1 / 4$ \\
\hline BUN levels (mg/dL) (average \pm SEM) & $20 \pm 1.53$ & $26.5^{*} \pm 1.42$ & $14.7 \pm 1.48$ & $13 \pm 1.70$ & $16 \pm 2.04$ & $23.5^{\star} \pm 2.44$ \\
\hline Platelet levels $\left(\times 10^{3} / \mu \mathrm{L}\right)$ (average \pm SEM) & $357 \pm 0.03$ & $375.8 \pm 0.04$ & $342 \pm 0.04$ & $310.5 \pm 0.04$ & $205^{\star} \pm 0.04$ & $162^{\star} \pm 0.04$ \\
\hline
\end{tabular}

${ }^{*} p$-value $\leq 0.05$ compared to day 0 ; average \pm standard error of predicted mean is shown. 


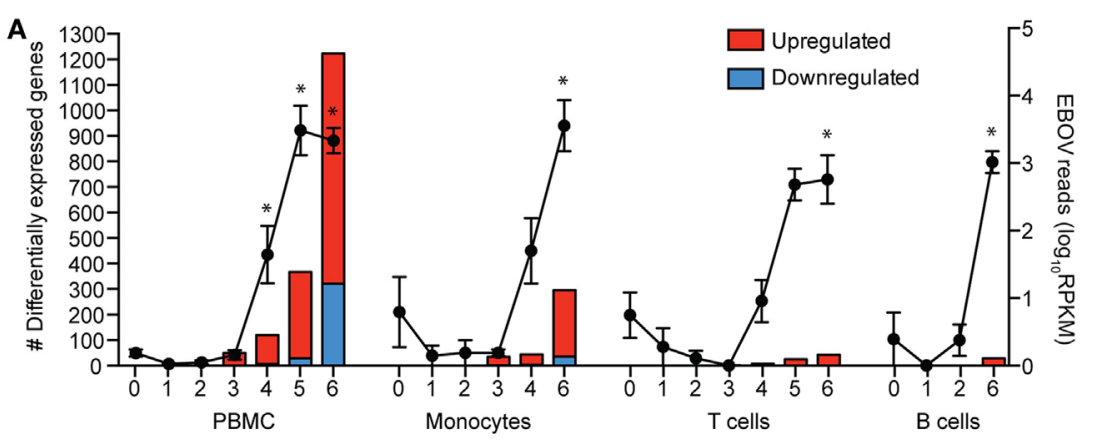

Days post infection

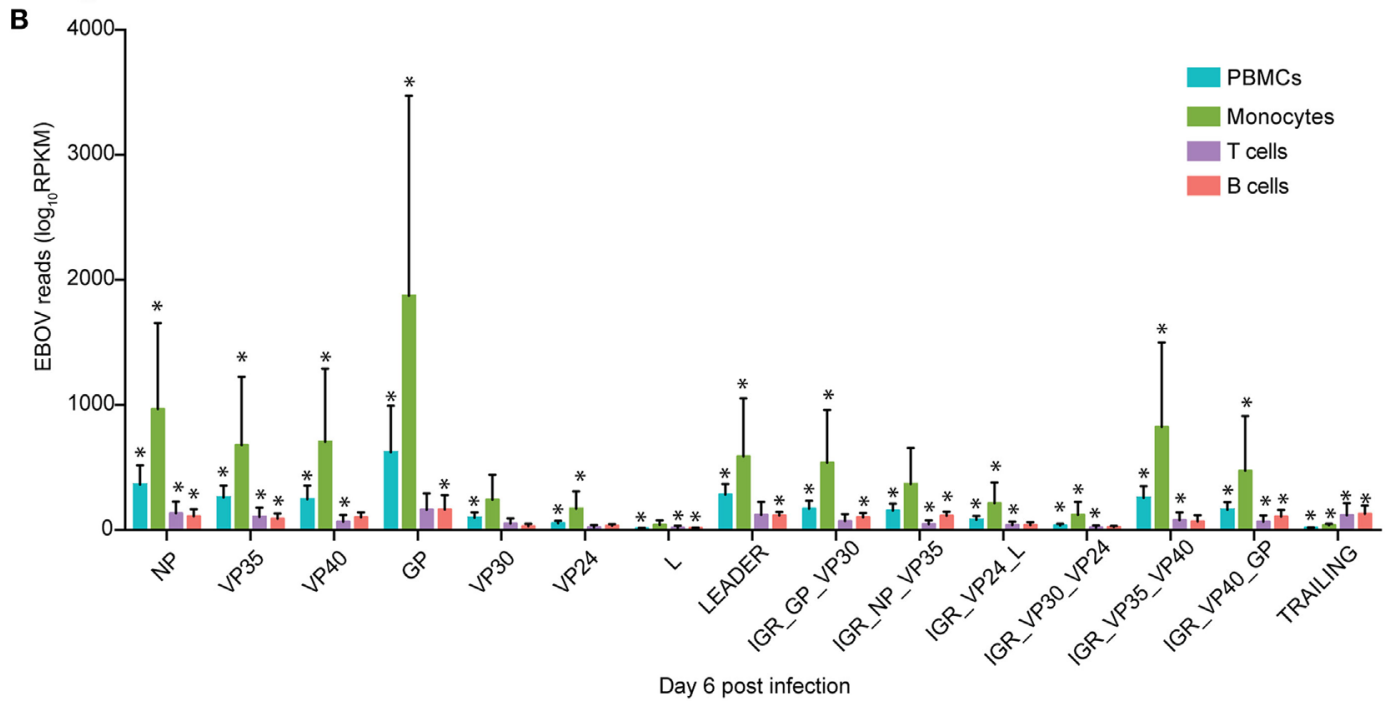

FIGURE 1 | Monocytes support ZEBOV-Makona replication and are major contributors of inflammation. (A) Bar graph depicts number of protein coding differentially expressed genes (DEGs; defined as those $\geq 2$ fold change and false discovery rate (FDR) corrected $p$-value $\leq 0.05$ compared to 0 DPI) that have human homologs (red indicates upregulated while blue indicates downregulated DEGs) in peripheral blood mononuclear cells (PBMC), monocytes, T cells, and B cells. Line graph represents number of normalized viral transcripts (RPKM); mean \pm SEM are shown; * $p \leq 0.05$ compared to day 0 . (B) RPKM normalized RNA sequencing reads mapping to each ZEBOV open reading frame (ORF), intergenic region (IGR), as well as leader and trailing sequences 6 DPI within PBMC, monocytes, T-cells, and B-cells. FDR adjusted $p$-value was obtained following EdgeR analysis; mean \pm SEM are shown; ${ }^{*} p \leq 0.05$ compared to day 0 .

1-3) (Figure 2C). Also upregulated, were STAT1 and 2 (Signal Transducer and Activator of Transcription 1 and 2), which serve as transcriptional activators of ISGs (37). Other DEGs of note that were upregulated throughout infection include the monocyte chemoattractant CXCL10 (C-X-C Motif Chemokine Ligand 10) and the C1 inhibitor SERPING1 (Serpin Family G Member 1). Additional ISGs such as IFIT5, IFIT1B, and ISG15 [Interferon, Alpha-Inducible Protein (Clone IFI-15K)] as well as genes involved in receptor-mediated endocytosis such as SIGLEC1 (Sialic Acid Binding Ig-Like Lectin 1) (38) were upregulated 4 and 6 DPI. In contrast, expression of adhesion molecules NCAM1 (Neural Cell Adhesion Molecule 1), AMICA (Adhesion Molecule Interacting with CXADR Antigen 1), and SPECC1 (Sperm Antigen with Calponin Homology and Coiled-Coil Domains) and transcription factor $A H R$ (Aryl Hydrocarbon Receptor) were downregulated 4-6 DPI (Figure 2C).

A large number of DEGs were identified 6 DPI (Table S2 in Supplementary Material). Upregulated DEGs enriched to GO terms related to defense response/inflammation, oxidative stress, and apoptosis (Figure 2B). Of the 146 DEGs that enriched to GO term "Immune system process," 98 directly interacted with each other (Figure 3A). Expression of these genes was regulated by STAT1, STAT2, IRF7 (Interferon Regulatory Factor), and $C E B P B$ (CCAAT/Enhancer Binding Protein, Beta). Downstream mediators of these transcription factors are involved in: leukocyte recruitment, e.g., CCL2 and CCL4L1 (39, 40); signaling, e.g., IRAK2 (Interleukin 1 Receptor Associated Kinase 2) (41) and CSF3R (Colony stimulating factor 3 receptor); and pathogen recognition, e.g., TLR2 and MYD88. Other upregulated genes in this network play a role in: coagulation such as THBS1 (Thrombospondin 1) (42); apoptosis such as BCL2A1 (BCL2 Related Protein A1) (43); response to oxidative stress, e.g., SOD2 (Superoxide dismutase 2) (44); positive regulation of nitric oxide synthesis such as GCH1 (GTP Cyclohydrolase 1) (45); and vascular integrity, e.g., MMP9 (matrix metalloproteinase-9) (46). Upregulated genes that also enriched to "Immune system process" 
TABLE 2 | Functional enrichment of differentially expressed genes (DEGs) in peripheral blood mononuclear cells 6 DPI.

\begin{tabular}{lcr}
\hline Gene Ontology & $\begin{array}{c}\text { False discovery rate } \\
\text { adjusted } \boldsymbol{p} \text {-value }\end{array}$ & $\begin{array}{c}\text { Number of } \\
\text { DEGs }\end{array}$ \\
\hline Upregulated & & 358 \\
Immune system process & $3.02^{-82}$ & 255 \\
Defense response & $2.19^{-63}$ & 418 \\
Response to stress & $2.73^{-61}$ & 154 \\
Innate immune response & $7.57^{-47}$ & 173 \\
Response to cytokine & $1.34^{-45}$ & 117 \\
Inflammatory response & $1.61^{-34}$ & 158 \\
Cell death & $7.53^{-32}$ & 436 \\
Signal transduction & $9.15^{-29}$ & 312 \\
Positive regulation of metabolic process & $1.07^{-28}$ & 47 \\
Myeloid leukocyte activation & $3.05^{-23}$ & 103 \\
Downregulated & & 40 \\
Regulation of signal transduction & $6.16^{-10}$ & 90 \\
Leukocyte activation & $8.85^{-10}$ & 34 \\
Immune system process & $1.51^{-9}$ & 44 \\
Lymphocyte activation & $1.08^{-8}$ & 104 \\
Regulation of cell motility & $6.82^{-11}$ & 25 \\
Positive regulation of metabolic process & $1.06^{-7}$ & 13 \\
Adaptive immune response & $9.93^{-7}$ & \\
Regulation of blood coagulation & $2.49^{-6}$ & \\
\hline & & \\
\hline
\end{tabular}

but not included in the network include: CD274 (PD-L1), which inhibits T-cell activation (47); neutrophil chemoattractant IL8 (48); and monocyte chemoattractant CCL8 (Monocyte chemotactic protein 2) (49). Notable upregulated genes that play a role in apoptosis and oxidative stress include DDIT3 (DNA Damage Inducible Transcript 3) (50), HSPB1 (Heat shock protein Family B Member 1) (51) CASP5 (Caspase 5) (52), and BIRC3 (Baculoviral IAP Repeat Containing 3) (53) (Figure 3B).

The 36 genes downregulated 6 DPI enriched to metabolic and immune processes (Figure 2B). Some of these DEGs that enriched to "Antigen processing and presentation of peptide antigen via MHC class II" include AP1S2 (Adaptor Related Protein Complex 1 Sigma 2 Subunit), DNM1 (Dynamin 1), OSBPL1A (Oxysterol Binding Protein Like 1A), CD74 (Major histocompatibility Complex, Class II), and LGMN (Legumain) (Figure 3C). We also observed downregulation of transcription factors $A H R$ (Aryl Hydrocarbon Receptor), FOSB (FBJ Murine Osteosarcoma Viral Oncogene), and KLF4 (Kruppel-Like Factor). Finally, genes involved in translation, i.e., EIF3L (Eukaryotic Translation Initiation Factor 3 Subunit L), RPS3A, and RPS4Y2 (Ribosomal Proteins S3 and S4 Y-Linked 2) were also downregulated (Figure 3C).

To investigate the association between transcriptional changes detected within monocytes and systemic inflammation, we performed a Spearman correlation between plasma levels of cytokines and chemokines and RPKM values of DEGs detected in monocytes 6 DPI. We observed strong correlations $(R \geq 0.8)$ between DEGs detected within monocytes 6 DPI and circulating inflammatory cytokines IL1 $\beta$ (42 DEGs), IL6 (53 DEGs), IFN $\alpha$ (92 DEGs), IL7 (40 DEGs), IFN $\gamma$ (40 DEGs), IL18 (74 DEGs), and IL4 (45 DEGs) as well as chemokines MIP1 $\alpha$ (110 DEGs), and MCP1 (45 DEGs) (Figure S4 in Supplementary Material).

\section{ZEBOV-Makona Infection Induces Limited Differential Gene Expression in Lymphocytes}

To understand the consequence of ZEBOV-Makona infection on the adaptive immune response, we next determined transcriptional changes within $\mathrm{T}$ - and B-cells following challenge. The average purity of T-cells and B-cells was $>80 \%$ and $>65 \%$, respectively (Table S1 in Supplementary Material). In contrast to monocytes, limited host and viral transcriptional changes were detected in T-cells (Figures $\mathbf{1 A}$ and $\mathbf{4 A}$ ). In addition, DEGs detected in T-cells were primarily involved in "Type I IFN signaling" (Figure 4B) and consisted of ISGs that play a role in antiviral immunity, i.e., DHX58, GBP1, IFIT1/2, OAS1-2, and MX1 (Figure 4C). Last, PHF11 (PHD Finger Protein 11) and TREML2 (Triggering Receptor Expressed on Myeloid Cells Like 2), both of which are involved in T-cell activation $(54,55)$, were also upregulated 6 DPI. Similarly, we detected a limited number of gene expression changes (31 DEGs) in B-cells 6 DPI (Figure 1A), which also enriched to GO terms associated with innate immune defense to viral infection (Figure 5A) and consisted primarily of ISGs, i.e., DHX58, GBP1, IFIT1-3, 5 (Figure 5B). Analysis of viral transcripts revealed that ZEBOV ORF and IGR transcripts in Tand B-cells were 2-12-fold lower compared to monocytes, with the exception of the trailing end region (Figure 1B). Euclidean distances also indicate that the viral transcript profile in $\mathrm{T}$ and $\mathrm{B}$ cells was more distant compared to that observed in PBMC (Figure S3 in Supplementary Material).

\section{Monocytes Contribute a Significant Proportion of Host Transcriptional Changes Detected in PBMC}

We next compared gene expression profiles of PBMC, monocytes, T-cells, and B-cells $6 \mathrm{DPI}$, the time point at which MakonaZEBOV infection caused the most significant clinical signs and transcriptional changes [Table 1 (26)]. Principle component analysis of normalized reads across all groups showed that monocytes were transcriptionally more similar to PBMC than T-cells and B-cells (Figure 6A). Comparison of all protein coding DEGs among the four groups revealed that $20 \%$ of DEGs detected in PBMC were also differentially expressed within monocytes. In contrast, the DEGs detected in T-cells and B-cells accounted for only 3.4 and $2.5 \%$ of the DEGs detected in PBMC (Figure 6B). As expected, the $24 \mathrm{DEG}$ common to all 4 groups play a role in Type I IFN response (Figure 6C). The 971 genes that were only differentially expressed in PBMC had lower fold changes and their expression did not reach the statistical cutoff of FDR adjusted $p$-value $\leq 0.05$ and average $\mathrm{RPKM} \geq 5$ in each individual cell subset (Figure 6D). The 250 DEGs detected in both monocytes and PBMC enriched to GO terms linked to immunity and cell death (Figure 7A). Functional enrichment of the 971 genes exclusive to PBMC are involved in similar biological processes as the 250 DEGs found in monocytes related to host defense, innate immune response, and inflammation (Figure 7B). These data suggest that these DEGs are only significant when transcriptional changes across all subsets are included in the analysis. The 46 DEGs detected exclusively in monocytes 6 DPI mapped to GO 

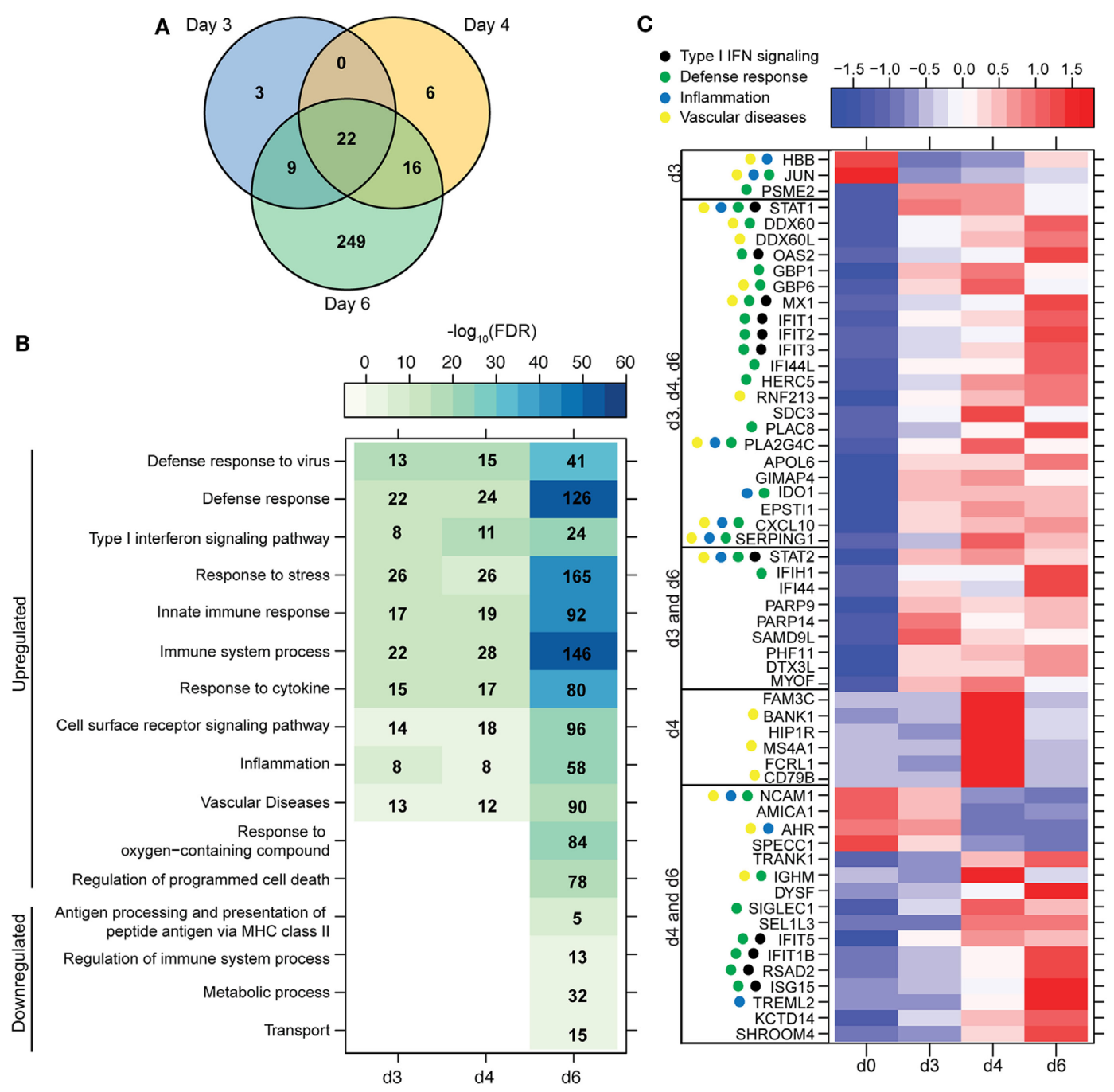

FIGURE 2 | ZEBOV-Makona infection induces expression of genes involved in defense response and Type I IFN signaling in monocytes. (A) 3 way Venn diagram displaying overlap between differentially expressed genes (DEGs) detected 3, 4, and 6 DPI in purified monocytes. (B) Heatmap representing functional enrichment of DEGs 3, 4, and 6 DPI; color intensity represents the statistical significance (shown as negative $\log _{10}$ of the false discovery rate (FDR)-corrected $p$-value); range of colors is based on the lowest and highest $-\log _{10}($ FDR $)$ values for the entire set of terms; the number of DEGs mapping to each functional enrichment term each day is listed within each box; blank boxes represent no statistical significance. (C) Heatmap representing gene expression (shown as absolute normalized RPKM values) of all DEGs detected 3 and 4 DPI; genes enriching to Gene Ontology or disease term are indicated by colored dot; range of colors is based on scaled and centered RPKM values of the entire set of genes (red represents increased expression while blue represents decreased expression); each column represents the median RPKM values for each DPI.

terms such as "Innate immune response" and "Antigen processing and presentation of exogenous peptide antigen" and had higher magnitude of expression compared to PBMC, suggesting that their expression was diluted by the inclusion of the additional subsets in PBMC analysis (Figures 7C,D).

\section{DISCUSSION}

In this manuscript, we report the first in vivo longitudinal analysis of viral and host transcriptional changes in purified monocytes, T-cells, and B-cells isolated from cynomolgus macaques following ZEBOV-Makona infection. The susceptibility of monocytes to $\mathrm{ZEBOV}$ infection has been controversial. Some in vitro studies showed that filoviruses (MARV, ZEBOV) can replicate in both primary monocytes and MDMs with similar virus growth kinetics $(6,7,15)$, whereas other in vitro studies reported delayed ZEBOV entry in undifferentiated monocytes compared to macrophages (17). Similarly, PMA-naïe THP-1 cells were resistant to virus like particles pseudotyped with ZEBOV GP and become permissive only following differentiation (17). Our data strongly suggest circulating blood monocytes are permissive to ZEBOV replication in vivo given the significant increase in $\mathrm{ZEBOV}$ reads 6 DPI. Furthermore, as previously reported in Vero E6 cells, NP was one of the most transcribed genes, whereas L 
was the least transcribed (56). These observations are in line with earlier studies reporting viral antigens within DC and monocytes in draining lymph nodes following intramuscular challenge with EBOV (4). In support of the ability of monocytes to permit viral replication, genes involved in receptor-mediated endocytosis 4 DPI (SIGLEC1 and HIP1R) as well as Cathepsin $\mathrm{L}$, which plays a role in ZEBOV-GP mediated entry (57), were upregulated 6 DPI.

Differentially expressed genes were detected as early as 3 DPI in monocytes, confirming they are among the early targets of ZEBOV infection (4). Although we did not detect increased transcripts of IFN $\alpha / \beta$ within monocytes, we saw increased expression of ISGs (e.g., IFIT1-4, OAS1-2, OASL, RSAD2, ISG15, and GBP1). Similarly, ISGs (IFIT1-2, GBP1, $O A S L)$ have been detected in MDMs $6 \mathrm{~h}$ post in vitro infection with ZEBOV-Mayinga (EBOV strain isolated in 1976) in the absence of increased IFN $\alpha / \beta$ expression (58). A possible reason for elevated ISGs in our study is that monocytes may be responding to low levels of IFN $\alpha / \beta$ secreted from non-infected
DCs and monocytes that are activated by viral debris, such as "shed" GP (59). Moreover, it has been previously shown that ZEBOV virion binding mediated by GP is responsible for early macrophage transcriptional responses before the detection of viral replication (58). A second possible explanation is that the increased expression of ISGs is independent of the activation of canonical Type I IFN signaling. Pulit-Penaloza et al. reported the upregulation of OAS1a, OAS1b, IRF1, and IRF7 in STAT $1^{-/-}$, STAT2 $2^{-/-}$, and IFN $\alpha / \beta$ receptor $^{-/-}$mouse embryonic fibroblasts infected with West Nile Virus (60), indicating that an alternative activation mechanism exists for inducing ISG expression in the absence of Type I IFN signaling.

We observed the most transcriptional changes in ZEBOVMakona infected monocytes 6 DPI. A large number of genes upregulated $6 \mathrm{DPI}$ are important for inflammatory responses such as CXCL10, SERPING1, PPBP, IL8, CSF3R, IL1R1, MYD88, $I R A K 2$, and NFKBIA. The increased expression of chemokines CCL2, CCL4L1, and CCL8 may be important for the recruitment of additional monocytes and DCs to sites of infection allowing

A

Immune system process

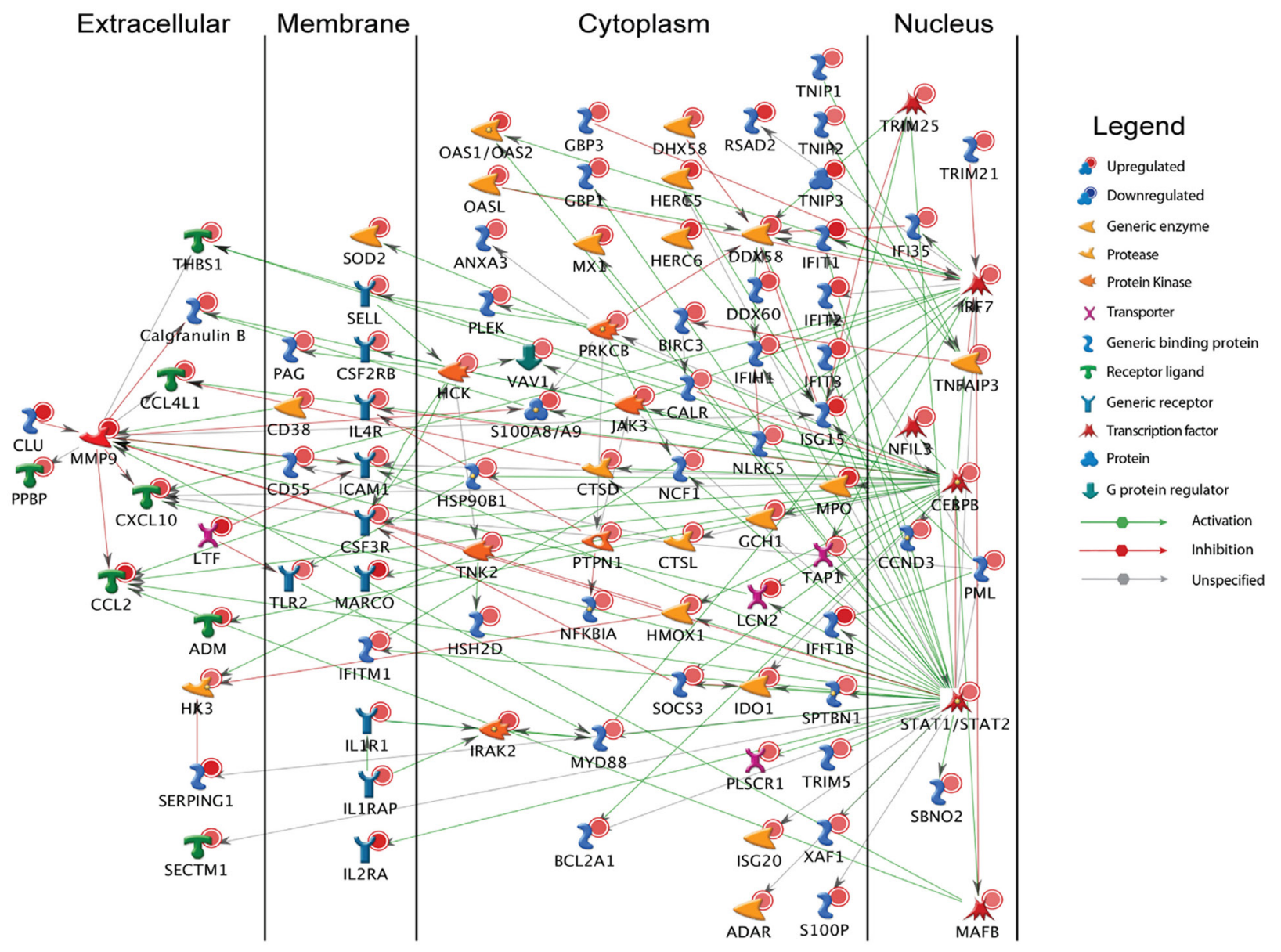

FIGURE 3 | Continued 


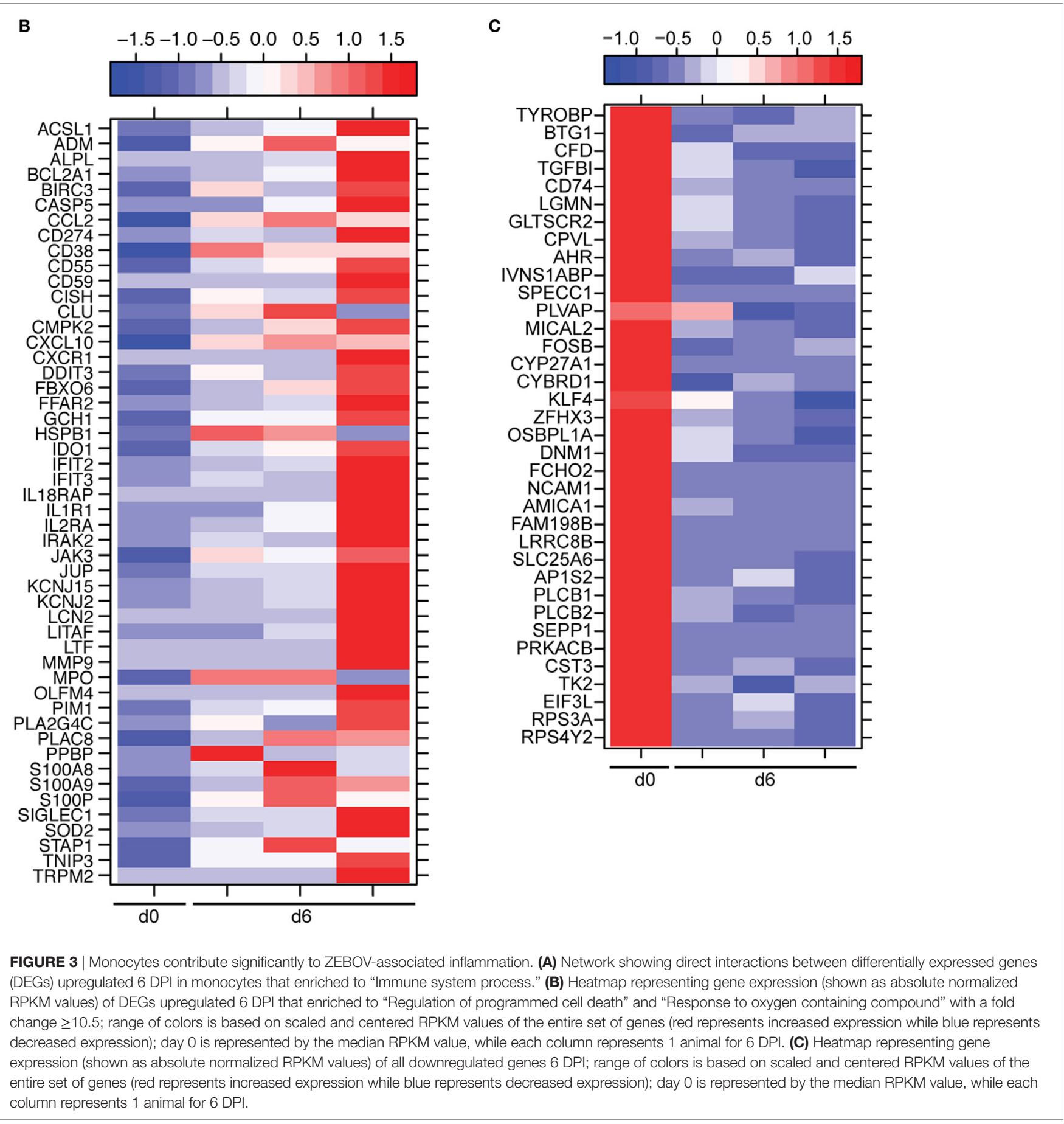

ZEBOV to disseminate. Furthermore, additional analysis revealed a strong association between DEGs detected in monocytes 6 DPI to circulating levels of inflammatory immune mediators including IFN $\alpha$, IL-6, MIP-1 $\alpha$, and MCP-1 (CCL2). Overall, there was strong agreement between the type of association (positive or negative) with immune mediators and observed changes in gene expression. For instance, expression of most ISGs were positively associated with IFN $\alpha$. Although some genes were predicted to be negatively regulated by IL1 $\beta$ and IL18, their expression increased, suggesting that they are positively regulated by other mediators not included in this analysis.

Expression of genes involved in apoptosis (OLFM4, BCL2A1, $C A S P 5$, and BIRC3) and unfolded protein response (DDIT3, FBX06, HSPB1, and HSPA5) were significantly increased. Interestingly, HSPB1 and HSPA5 proteins have been identified as EBOV associated host factors, and targeting of HSPA5 in vivo 
A

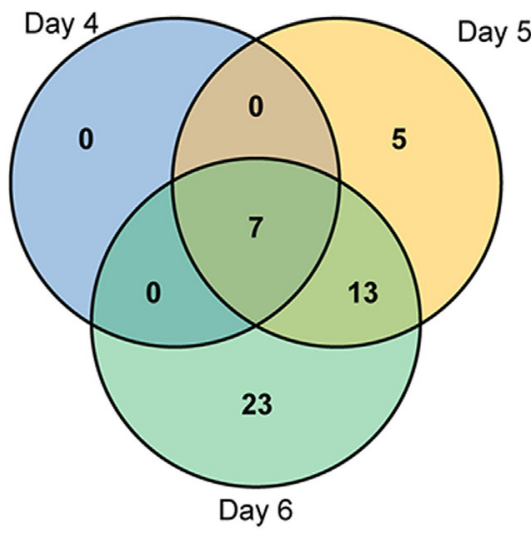

B

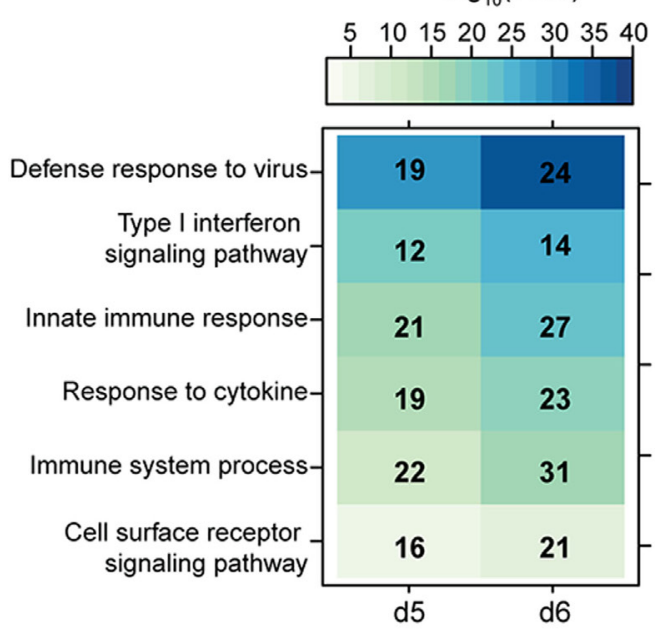

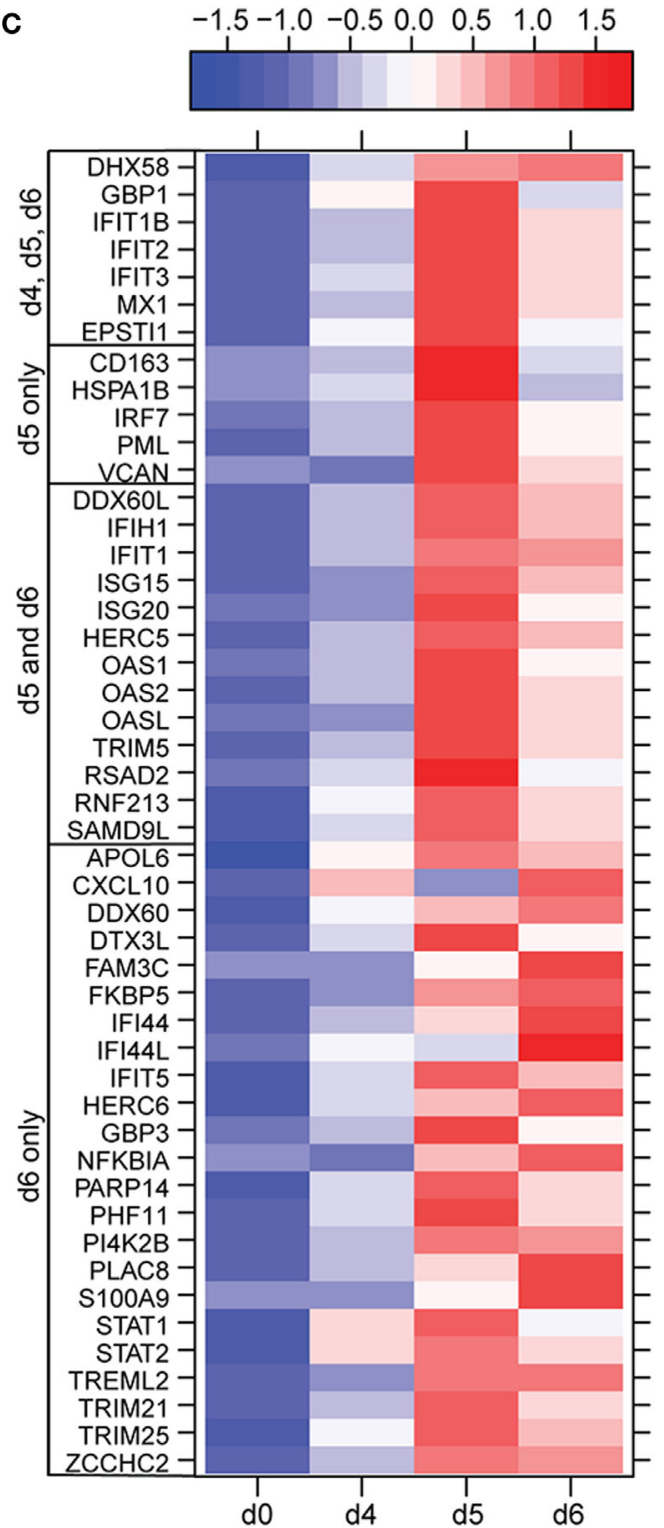

C

FIGURE 4 | ZEBOV-Makona infection induces limited differential gene expression in T-cells. (A) 3 way Venn diagram displaying overlap between differentially expressed genes (DEGs) detected 4, 5, and 6 DPI. (B) Heatmap representing functional enrichment of DEGs 5 and 6 DPI; color intensity represents the statistical significance (shown as negative $\log _{10}$ of the false discovery rate (FDR)-corrected $p$-value); range of colors is based on the lowest and highest -log ${ }_{10}(\mathrm{FDR}$ ) values for the entire set of terms; the number of DEGs mapping to each Gene Ontology term each day is listed within each box. (C) Heatmap representing gene expression (shown as absolute normalized RPKM values) of all DEGs detected 4, 5, and 6 DPI; range of colors is based on scaled and centered RPKM values of the entire set of genes (red represents increased expression while blue represents decreased expression); each column represents the median RPKM values for each DPI.

using PMOs in mice before challenge increased survival rates $(61,62)$. Additionally, we identified an increase in transcripts associated with the production of reactive nitric/oxygen species (GCH1 and SOD2) and vascular function (ADM, MMP9, THBS1, and SERPINA1). This is consistent with documented reports of increased nitric oxide in plasma of fatal cases compared to patients who survived (10). Furthermore, increased expression of $M M P 9$, as well as other metalloproteinase genes, in the spleen of mice challenged with mouse adapted (MA)-ZEBOV have been associated with lethality (63). Interestingly, we did not detect changes in tissue factor (TF) transcripts, which has previously been reported to increase in lymphoid macrophages collected from EBOV challenged NHPs (16). However, TF is produced by tissue macrophages, therefore transcriptional analysis of circulating monocytes may have precluded us from observing changes in TF. In line with a recent study that revealed increased numbers of T-cells expressing inhibitory molecule PD-1 in ZEBOV-Makona fatal cases (64), we detected an upregulation of CD274 (PD-L1), 

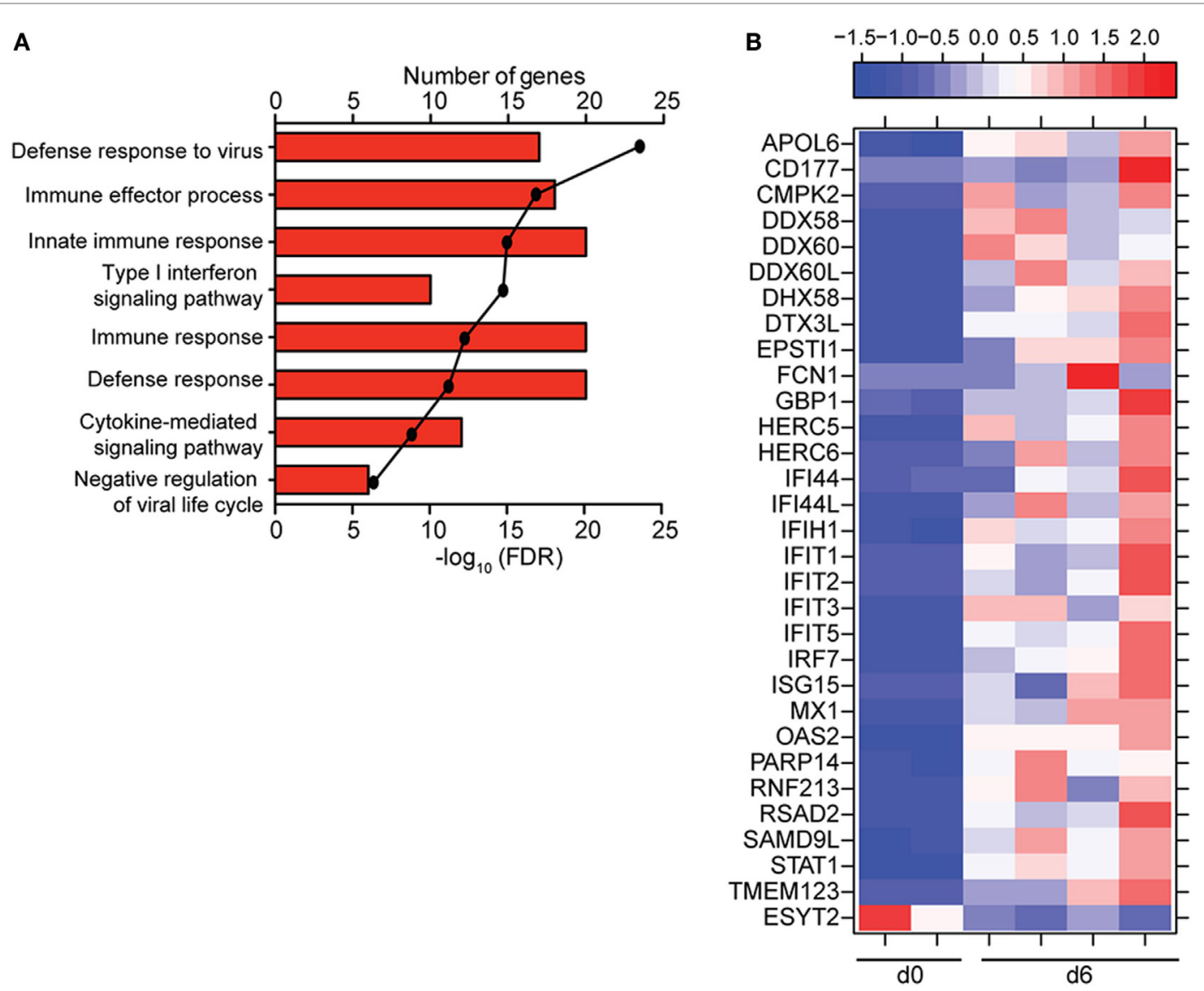

FIGURE 5 | ZEBOV-Makona infection induces limited differential gene expression in B-cells. (A) Functional enrichment of upregulated genes detected 6 DPI; horizontal bar graphs represent number of genes that mapped to each Gene Ontology term listed while line graph represents false discovery rate (FDR)-corrected p-value. (B) Heatmap representing gene expression (shown as absolute normalized RPKM values) of all differentially expressed genes 6 DPI; range of colors is based on scaled and centered RPKM values of the entire set of genes (red represents increased expression while blue represents decreased expression); each column represents 1 animal.

which inhibits TCR-mediated activation. Although it did not reach our statistical cutoff, we did detect an increase in PD-1 transcripts (fold change of 3.29) 6 DPI in T-cells. In contrast, genes associated with antigen processing and presentation (AP1S2, OBPL1, CD74, LGMN) were downregulated. Collectively, these data suggest that infected monocytes and macrophages are likely to be one of the major drivers of excessive inflammation during ZEBOV infection and provide insight into the failure of initiating an adaptive immune response. We also detected a downregulation of genes associated with translation, including genes encoding for translation initiation factors and ribosomal proteins, a strategy that may be employed by the host to limit ZEBOV replication and spread.

Our observations differ from an in vitro study by Wahl-Jensen et al. where they identified 88 DEGs in macrophages within the first $6 \mathrm{~h}$ after ZEBOV-Mayinga infection (58). The earlier detection of DEGs in the Wahl-Jensen study correlates with the faster onset of clinical symptoms seen after Mayinga infection compared to Makona (27). Additionally, the earlier detection of DEGs could be due to the high multiplicity of infection used in vitro that may not be reached until later time points during in vivo infection. Of the 88 DEGs identified in the Wahl-Jensen study, 20 were common with our data set including genes involved in: apoptosis (TNFAIP3, BIRC3); immune defense, inflammation, and chemotaxis (TNFAIP6, TX3, IL8, CCL8, PPBP, TRIM25, and IL15RA); adhesion (ICAM1); oxidative stress (SOD2, GCH1); and ISGs (IFIT1-2, OASL, GBP1). In line with our findings, a recent study by Liu et al. showed that patients who succumbed to ZEBOV-Makona infection had a stronger upregulation of acute phase responses and an increased abundance of ISG transcripts compared to survivors (65). Moreover, longitudinal transcriptomic analysis in peripheral blood from a surviving male health care worker exposed to EBOV during the 2013-2016 ZEBOVMakona epidemic revealed that transcripts that positively correlated with viremia enriched to GO terms associated with activation of antiviral responses (including Type I IFN signaling), inflammation, oxidative stress, DNA damage and cell death (66). Additionally, transcripts with a positive correlation with D-dimer levels and negative correlation with platelet levels functionally enriched to processes associated with macrophage and 


\section{A}

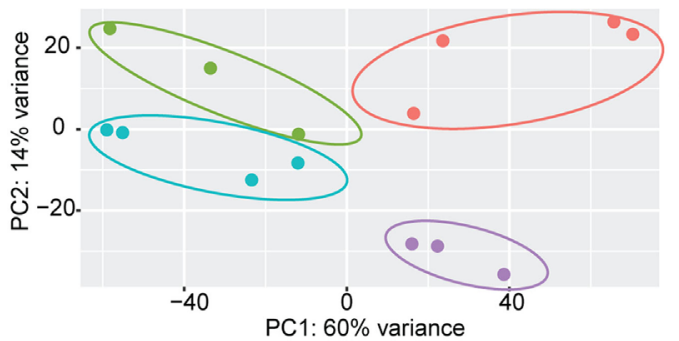

C

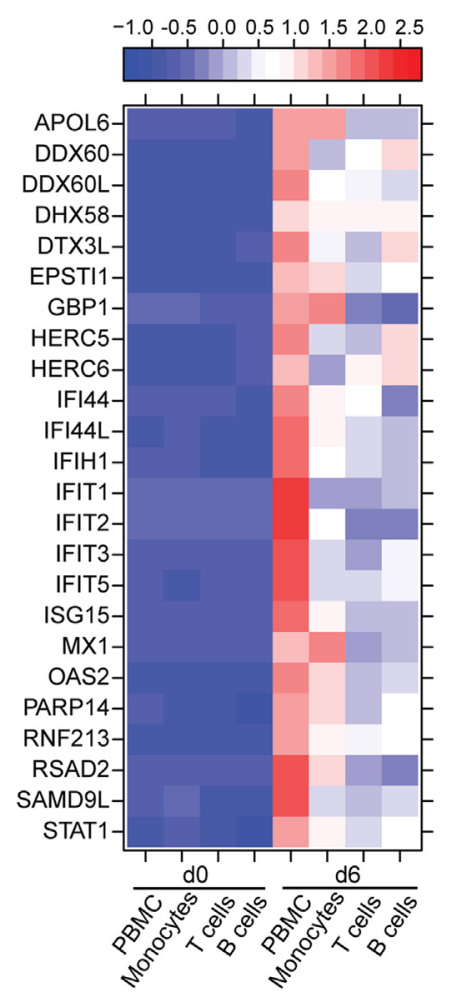

B

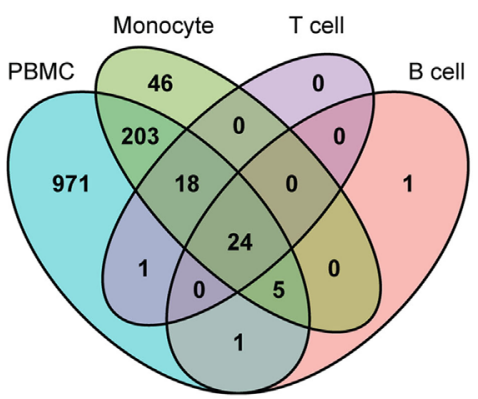

D D
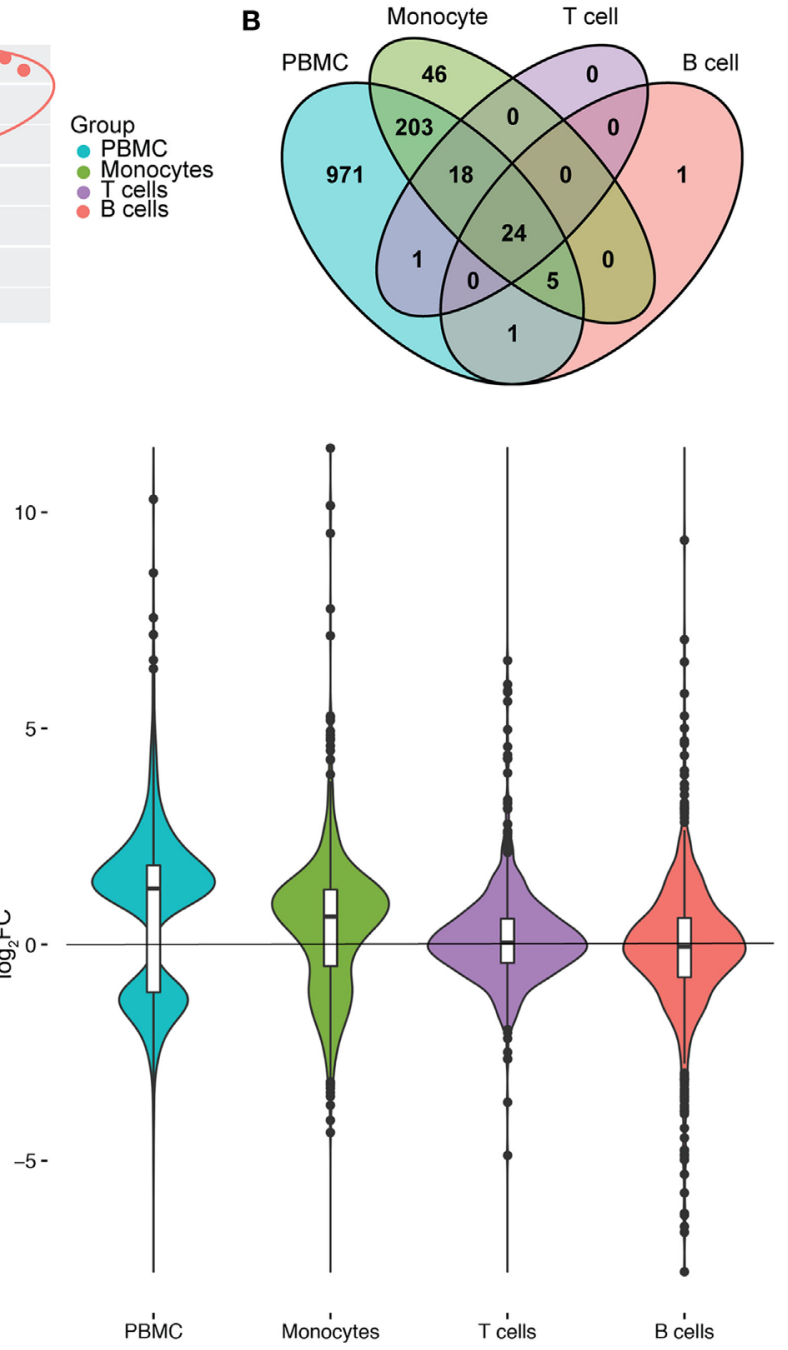

FIGURE 6 | Monocytes contribute a significant proportion of transcriptional changes detected in peripheral blood mononuclear cells (PBMC). (A) Principal component analysis of normalized transcript numbers 6 DPI in PBMC, monocytes, T-cells, and B-cells. (B) 4 way Venn diagram showing overlap between differentially expressed genes (DEGs) detected 6 DPI in PBMC, monocyte, T-cells, and B-cells. (C) Heatmap representing gene expression (shown as absolute normalized RPKM values) of the 24 DEGs detected in PBMC as well as purified monocytes, T- and B-cells 6 DPI; range of colors is based on scaled and centered RPKM values of the entire set of genes (red represents increased expression while blue represents decreased expression); each column represents the median RPKM values on each day for each subset. (D) Violin plot of the $\log _{2} F C$ of 971 DEGs that were exclusively detected in PBMC within each cell subset.

neutrophil recruitment, macrophage death, antiviral response, and phagocytosis (66).

Additional bioinformatics analysis revealed that $62 \%$ of DEGs detected within monocytes 6 DPI were shared with the transcriptional changes in MDMs and macrophages differentiated from human induced pluripotent stem cells (IPSDMs) following $6 \mathrm{~h}$ LPS stimulation (Figure S5A in Supplementary Material) (29). These common genes enriched to GO terms associated with inflammation, response to cytokine, and response to Type I interferon (Figure S5B in Supplementary Material). This observation suggests that ZEBOV infection may result in monocyte activation. In contrast, the $40 \%$ of DEGs that were unique in our data set enriched to wounding, coagulation, hemostasis, and response to oxidative stress, which is in line with the development of EVD (Figure S5C in Supplementary Material).

In contrast to monocytes, our data indicate that $\mathrm{T}$ - and B-cells are less permissible to ZEBOV replication, as evidenced by the lower number of viral transcripts in lymphocytes. Additionally, the abundance of viral transcripts was comparable among all ZEBOV ORFs, suggesting abortive transcription. This is consistent with previous in vivo and in vitro reports that show ZEBOV 

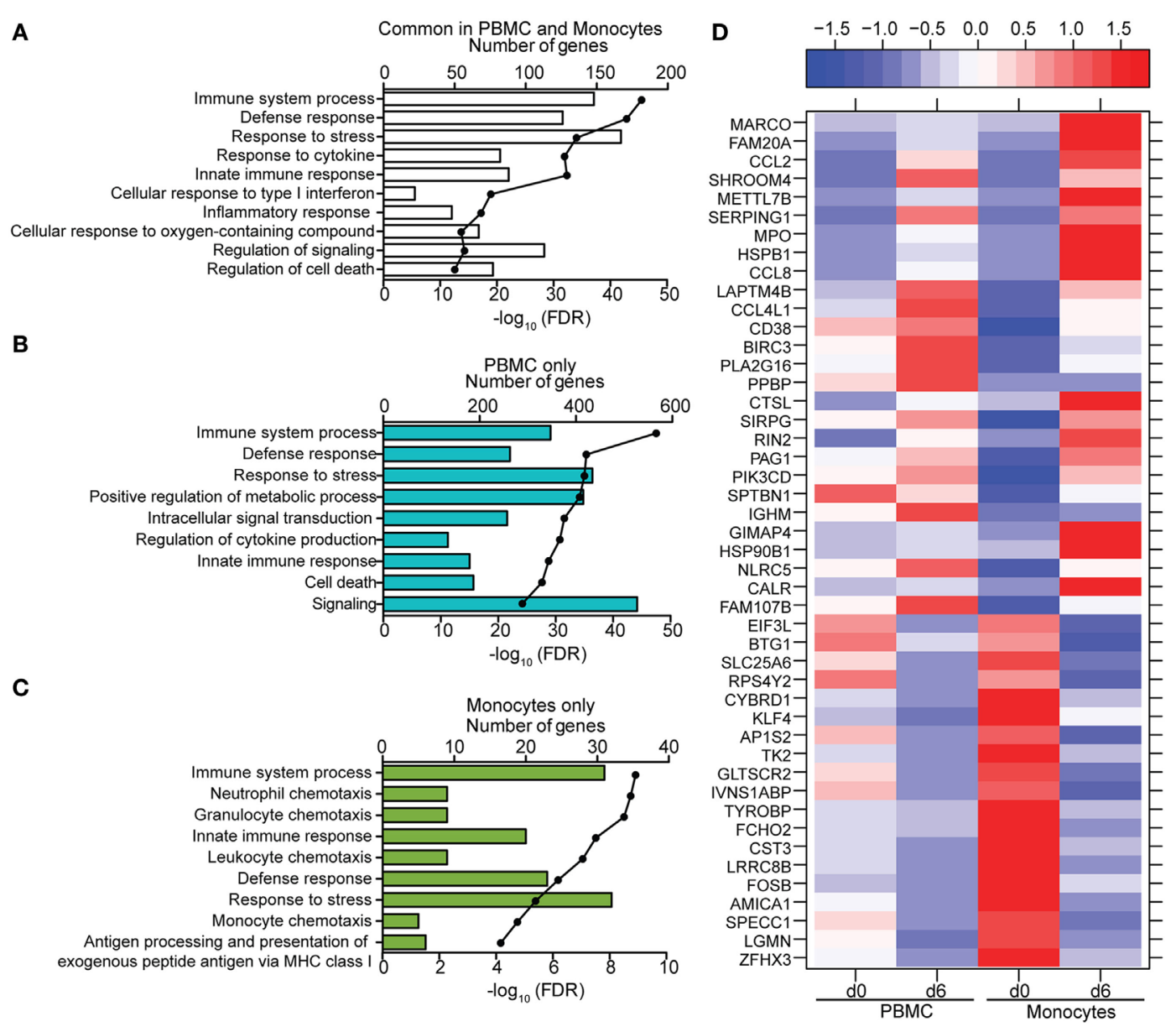

FIGURE 7 | Comparison of transcriptional profiles between peripheral blood mononuclear cells (PBMC) and monocytes 6 DPI. (A-C) Functional enrichment of differentially expressed genes (DEGs) detected in both monocytes and PBMC (A), PBMC only (B), or monocytes only (C); horizontal bar graphs represent number of genes that mapped to each Gene Ontology term listed while line graph represents false discovery rate (FDR)-corrected $p$-value. (D) Heatmap representing gene expression (shown as absolute normalized RPKM values) of the 46 DEGs that were only detected in monocytes 6 DPI; corresponding RPKM values in PBMC are shown for comparison; range of colors is based on scaled and centered RPKM values of the entire set of genes (red represents increased expression while blue represents decreased expression); each column represents the median RPKM values on each day for each subset.

does not replicate within lymphocytes and that lymphocyte apoptosis is likely an indirect result of ZEBOV induced upregulation of negative regulators and inflammatory mediators $(15,23,24)$. The low number of ZEBOV reads detected in T- and B-cells 6 DPI may be due to increased amounts of circulating fibrin bound to cells and viral particles resulting in viral genome carry-over during cell separation.

The small number of DEGs detected in T and B cells 6 DPI were overwhelmingly ISGs. This increase in ISG expression within lymphocytes correlates with the large increase in circulating IFN $\alpha$ levels observed 6DPI. However, fold changes of ISG expression were much lower in lymphocytes compared to monocytes. Previous experiments have demonstrated that IFN $\alpha$ subtypes can activate the JAK/STAT pathway and induce ISGs in T-cells albeit at a lower magnitude compared to DCs (67). Interestingly, we did not detect changes in the expression of genes that play a role in lymphocyte apoptosis such as FAS, caspase, and TRAIL despite the lymphopenia we observed in vivo. In contrast, our previous analysis of transcriptional changes within blood samples isolated from the same animals showed a large decrease in expression of lymphocyte related genes and a robust increase in expression of pro-apoptotic genes (26). These observations strongly suggest that the absence of such transcripts in this study is due to the fact that T- and B-cells purified from PBMC, are mostly viable cells not undergoing cell death. Importantly, we were unable to detect any signatures of an adaptive immune response, such as antiviral cytokine IFN $\gamma$ or cytolytic molecules perforin and granzyme B, needed to clear viral infection and establish memory. A recent study showed production of immunosuppressive mediators IL-10 and PD-L1 is dependent on Type I IFN signaling in a mouse model of lymphocytic choriomeningitis virus infection $(68,69)$. Therefore, continuous Type I IFN response, including sustained expression of ISGs, driven by ZEBOV-Makona infection may inhibit the ability of lymphocytes to develop into an effective defense response. One limitation in our study was the low purity of $B$ cells isolated at early time points post-infection. However, the 
transcriptional profile within $\mathrm{T}$ cells, which had very little contamination, was similar to B cells, suggesting any contamination had little influence on the gene expression changes we observed. Another limitation is that we were not able to determine transcriptional changes within DCs due to very low cell recovery following sequential isolation using magnetic beads given that this subset make up less than $1 \%$ of the PBMC population and was further decreased 6 DPI (26).

In line with the larger transcriptional changes observed, DEGs in monocytes contributed a more significant portion of transcriptional changes ( $20 \%$ compared to $2-3 \%)$ detected in PBMC 6 days post ZEBOV infection compared to T- and B-cells. The 250 common DEGs detected in the purified monocyte fraction and PBMC play a central role in immunity, inflammation, and cell death. However, 971 DEGs that were detected within total PBMC did not meet the criteria for DEGs within purified monocytes, T-, or B-cells. These 971 DEGs enriched to biological processes involved in innate as well as adaptive immunity. Therefore, it is possible that expression changes in these genes are only significant when combining changes in all three cell subsets. Conversely, by enriching for monocytes, changes in expression of genes important for chemotaxis and antigen presentation became more pronounced, likely because the transcription profile was more specific to these cells rather than diluted by the global PBMC transcriptional profile.

In summary, our data revealed that monocytes, but not lymphocytes, are supportive of ZEBOV replication. Our findings also indicate that while ZEBOV replication in monocytes may suppress IFN $\alpha / \beta$ mRNA expression $(7,70)$, it does not suppress ISG induction in infected cells. The gene expression patterns detected identify monocytes as one of the key players in ZEBOV

\section{REFERENCES}

1. Feldmann H, Sanchez A, Geisbert TW. "Filoviridae: Marburg and Ebola viruses," in Fields Virology. Vol. 1. 6th ed. Philadelphia, PA: Wolters Kluwer (2013).

2. Feldmann H, Geisbert TW. Ebola haemorrhagic fever. Lancet (2011) 377(9768):849-62. doi:10.1016/S0140-6736(10)60667-8

3. CDC. 2014 Ebola Outbreak in West Africa - Case Counts. (2016). Available from: http://www.cdc.gov/vhf/ebola/outbreaks/2014-west-africa/case-counts. html

4. Geisbert TW, Hensley LE, Larsen T, Young HA, Reed DS, Geisbert JB, et al. Pathogenesis of Ebola hemorrhagic fever in cynomolgus macaques: evidence that dendritic cells are early and sustained targets of infection. Am J Pathol (2003) 163(6):2347-70. doi:10.1016/S0002-9440(10)63591-2

5. Hensley LE, Young HA, Jahrling PB, Geisbert TW. Proinflammatory response during Ebola virus infection of primate models: possible involvement of the tumor necrosis factor receptor superfamily. Immunol Lett (2002) 80(3): 169-79. doi:10.1016/S0165-2478(01)00327-3

6. Stroher U, West E, Bugany H, Klenk HD, Schnittler HJ, Feldmann H. Infection and activation of monocytes by Marburg and Ebola viruses. J Virol (2001) 75(22):11025-33. doi:10.1128/JVI.75.22.11025-11033.2001

7. Gupta M, Mahanty S, Ahmed R, Rollin PE. Monocyte-derived human macrophages and peripheral blood mononuclear cells infected with Ebola virus secrete MIP-1alpha and TNF-alpha and inhibit poly-IC-induced IFN-alpha in vitro. Virology (2001) 284(1):20-5. doi:10.1006/viro.2001.0836

8. Baize S, Leroy EM, Georges AJ, Georges-Courbot MC, Capron M, Bedjabaga I, et al. Inflammatory responses in Ebola virus-infected patients. Clin Exp Immunol (2002) 128(1):163-8. doi:10.1046/j.1365-2249.2002.01800.x

9. Ebihara H, Rockx B, Marzi A, Feldmann F, Haddock E, Brining D, et al. Host response dynamics following lethal infection of rhesus macaques with Zaire pathogenesis by initiating events that contribute to characteristic symptoms of EVD including inflammation, vascular permeability, coagulation defects, and production of reactive oxygen species.

\section{ETHICS STATEMENT}

This study, including all protocols, was approved by the University of Texas Medical Branch at Galveston Institutional Animal Care and Use Committee in accordance with state and federal statutes and regulations relating to experiments involving animals and the Institutional Biosafety Committee.

\section{AUTHOR CONTRIBUTIONS}

IM and TG conceived and designed experiments. AM, KV, CW, CM, JG, RC, KA, and AJ conducted the experiments. All authors contributed to data analysis and interpretation, manuscript preparation, and final approval.

\section{FUNDING}

This work was supported by the National Institute of Allergy and Infectious Diseases, National Institutes of Health grant 5U19A109945.

\section{SUPPLEMENTARY MATERIAL}

The Supplementary Material for this article can be found online at http://www.frontiersin.org/article/10.3389/fimmu.2017.01372/ full\#supplementary-material.
Ebolavirus. J Infect Dis (2011) 204(Suppl 3):S991-9. doi:10.1093/infdis/ jir336

10. Sanchez A, Lukwiya M, Bausch D, Mahanty S, Sanchez AJ, Wagoner KD, et al. Analysis of human peripheral blood samples from fatal and nonfatal cases of Ebola (Sudan) hemorrhagic fever: cellular responses, virus load, and nitric oxide levels. J Virol (2004) 78(19):10370-7. doi:10.1128/JVI.78.19. 10370-10377.2004

11. Villinger F, Rollin PE, Brar SS, Chikkala NF, Winter J, Sundstrom JB, et al. Markedly elevated levels of interferon (IFN)-gamma, IFN-alpha, interleukin (IL)-2, IL-10, and tumor necrosis factor-alpha associated with fatal Ebola virus infection. J Infect Dis (1999) 179(Suppl 1):S188-91. doi:10.1086/514283

12. Wauquier N, Becquart P, Padilla C, Baize S, Leroy EM. Human fatal zaire Ebola virus infection is associated with an aberrant innate immunity and with massive lymphocyte apoptosis. PLoS Negl Trop Dis (2010) 4(10):e837. doi:10.1371/journal.pntd.0000837

13. Gupta M, Mahanty S, Bray M, Ahmed R, Rollin PE. Passive transfer of antibodies protects immunocompetent and imunodeficient mice against lethal Ebola virus infection without complete inhibition of viral replication. J Virol (2001) 75(10):4649-54. doi:10.1128/JVI.75.10.4649-4654.2001

14. Baize S, Leroy EM, Mavoungou E, Fisher-Hoch SP. Apoptosis in fatal Ebola infection. Does the virus toll the bell for immune system? Apoptosis (2000) 5(1):5-7. doi:10.1023/A:1009657006550

15. Geisbert TW, Hensley LE, Gibb TR, Steele KE, Jaax NK, Jahrling PB. Apoptosis induced in vitro and in vivo during infection by Ebola and Marburg viruses. Lab Invest (2000) 80(2):171-86. doi:10.1038/labinvest. 3780021

16. Geisbert TW, Young HA, Jahrling PB, Davis KJ, Kagan E, Hensley LE. Mechanisms underlying coagulation abnormalities in Ebola hemorrhagic fever: overexpression of tissue factor in primate monocytes/macrophages is a key event. J Infect Dis (2003) 188(11):1618-29. doi:10.1086/379724 
17. Martinez O, Johnson JC, Honko A, Yen B, Shabman RS, Hensley LE, et al. Ebola virus exploits a monocyte differentiation program to promote its entry. J Virol (2013) 87(7):3801-14. doi:10.1128/JVI.02695-12

18. Jaax NK, Davis KJ, Geisbert TJ, Vogel P, Jaax GP, Topper M, et al. Lethal experimental infection of rhesus monkeys with Ebola-Zaire (Mayinga) virus by the oral and conjunctival route of exposure. Arch Pathol Lab Med (1996) 120(2):140-55.

19. Baize S, Leroy EM, Georges-Courbot MC, Capron M, Lansoud-Soukate J, Debre P, et al. Defective humoral responses and extensive intravascular apoptosis are associated with fatal outcome in Ebola virus-infected patients. Nat Med (1999) 5(4):423-6. doi:10.1038/7422

20. Reed DS, Hensley LE, Geisbert JB, Jahrling PB, Geisbert TW. Depletion of peripheral blood T lymphocytes and NK cells during the course of Ebola hemorrhagic fever in cynomolgus macaques. Viral Immunol (2004) 17(3): 390-400. doi:10.1089/vim.2004.17.390

21. Geisbert TW, Young HA, Jahrling PB, Davis KJ, Larsen T, Kagan E, et al. Pathogenesis of Ebola hemorrhagic fever in primate models: evidence that hemorrhage is not a direct effect of virus-induced cytolysis of endothelial cells. Am J Pathol (2003) 163(6):2371-82. doi:10.1016/S0002-9440(10)63592-4

22. Gupta M, Spiropoulou C, Rollin PE. Ebola virus infection of human PBMCs causes massive death of macrophages, CD4 and CD8 T cell sub-populations in vitro. Virology (2007) 364(1):45-54. doi:10.1016/j.virol.2007.02.017

23. Connolly BM, Steele KE, Davis KJ, Geisbert TW, Kell WM, Jaax NK, et al. Pathogenesis of experimental Ebola virus infection in guinea pigs. J Infect Dis (1999) 179(Suppl 1):S203-17. doi:10.1086/514305

24. Wool-Lewis RJ, Bates P. Characterization of Ebola virus entry by using pseudotyped viruses: identification of receptor-deficient cell lines. JVirol (1998) 72(4):3155-60.

25. Yaddanapudi K, Palacios G, Towner JS, Chen I, Sariol CA, Nichol ST, et al. Implication of a retrovirus-like glycoprotein peptide in the immunopathogenesis of Ebola and Marburg viruses. FASEB J (2006) 20(14):2519-30. doi:10.1096/fj.06-6151com

26. Versteeg K, Menicucci AR, Woolsey C, Mire CE, Geisbert JB, Cross RW, et al. Infection with the Makona variant results in a delayed and distinct host immune response compared to previous Ebola virus variants. Sci Rep (2017) 7(1):9730. doi:10.1038/s41598-017-09963-y

27. Marzi A, Feldmann F, Hanley PW, Scott DP, Gunther S, Feldmann H. Delayed disease progression in cynomolgus macaques infected with Ebola virus Makona strain. Emerg Infect Dis (2015) 21(10):1777-83. doi:10.3201/ eid2110.150259

28. Backman TWH, Girke T. systemPipeR: NGS workflow and report generation environment. BMC Bioinformatics (2016) 17:388. doi:10.1186/ s12859-016-1241-0

29. Alasoo K, Martinez FO, Hale C, Gordon S, Powrie F, Dougan G, et al. Transcriptional profiling of macrophages derived from monocytes and iPS cells identifies a conserved response to LPS and novel alternative transcription. Sci Rep (2015) 5:12524. doi:10.1038/srep12524

30. Holm S. A simple sequentially rejective multiple test procedure. Scand J Statist (1979) 6(2):65-70.

31. Dong C, Davis RJ, Flavell RA. MAP kinases in the immune response. Annu Rev Immunol (2002) 20:55-72. doi:10.1146/annurev.immunol.20.091301.131133

32. Hofer TP, Zawada AM, Frankenberger M, Skokann K, Satzl AA, Gesierich W, et al. Slan-defined subsets of CD16-positive monocytes: impact of granulomatous inflammation and M-CSF receptor mutation. Blood (2015) 126(24):2601-10. doi:10.1182/blood-2015-06-651331

33. Grunin M, Hagbi-Levi S, Rinsky B, Smith Y, Chowers I. Transcriptome analysis on monocytes from patients with neovascular age-related macular degeneration. Sci Rep (2016) 6:29046. doi:10.1038/srep29046

34. Lacey DC, Achuthan A, Fleetwood AJ, Dinh H, Roiniotis J, Scholz GM, et al. Defining GM-CSF- and macrophage-CSF-dependent macrophage responses by in vitro models. J Immunol (2012) 188(11):5752-65. doi:10.4049/ jimmunol.1103426

35. Reiman JM, Kmieciak M, Manjili MH, Knutson KL. Tumor immunoediting and immunosculpting pathways to cancer progression. Semin Cancer Biol (2007) 17(4):275-87. doi:10.1016/j.semcancer.2007.06.009

36. Frankenberger M, Hofer TP, Marei A, Dayyani F, Schewe S, Strasser C, et al. Transcript profiling of CD16-positive monocytes reveals a unique molecular fingerprint. Eur J Immunol (2012) 42(4):957-74. doi:10.1002/eji.201141907
37. Schneider WM, Chevillotte MD, Rice CM. Interferon-stimulated genes: a complex web of host defenses. Annu Rev Immunol (2014) 32:513-45. doi:10.1146/annurev-immunol-032713-120231

38. O'Reilly MK, Paulson JC. Siglecs as targets for therapy in immune-cellmediated disease. Trends Pharmacol Sci (2009) 30(5):240-8. doi:10.1016/ j.tips.2009.02.005

39. Daly C, Rollins BJ. Monocyte chemoattractant protein-1 (CCL2) in inflammatory disease and adaptive immunity: therapeutic opportunities and controversies. Microcirculation (2003) 10(3-4):247-57. doi:10.1080/713773639

40. Modi WS. CCL3L1 and CCL4L1 chemokine genes are located in a segmental duplication at chromosome 17ql2. Genomics (2004) 83(4):735-8. doi:10.1016/j.ygeno.2003.09.019

41. Muralidharan S, Mandrekar P. Cellular stress response and innate immune signaling: integrating pathways in host defense and inflammation. J Leukoc Biol (2013) 94(6):1167-84. doi:10.1189/jlb.0313153

42. Bale MD, Mosher DF. Thrombospondin is a substrate for blood coagulation factor XIIIa. Biochem (1986) 25(19):5667-73. doi:10.1021/bi00367a048

43. Vogler M. BCL2A1: the underdog in the BCL2 family. Cell Death Differ (2012) 19(1):67-74. doi:10.1038/cdd.2011.158

44. Weisiger RA, Fridovich I. Mitochondrial superoxide dismutase - site of synthesis and intramitochondrial localization. JBiol Chem (1973) 248(13):4793-6.

45. McNeill E, Crabtree MJ, Sahgal N, Patel J, Chuaiphichai S, Iqbal AJ, et al. Regulation of iNOS function and cellular redox state by macrophage Gch1 reveals specific requirements for tetrahydrobiopterin in NRF2 activation. Free Radic Biol Med (2015) 79:206-16. doi:10.1016/j.freeradbiomed. 2014.10.575

46. Johnson C, Sung HJ, Lessner SM, Fini ME, Galis ZS. Matrix metalloproteinase- 9 is required for adequate angiogenic revascularization of ischemic tissues - potential role in capillary branching. Circ Res (2004) 94(2):262-8. doi:10.1161/01.RES.0000111527.42357.62

47. Butte MJ, Keir ME, Phamduy TB, Sharpe AH, Freeman GJ. Programmed death-1 ligand 1 interacts specifically with the B7-1 costimulatory molecule to inhibit T cell responses. Immunity (2007) 27(1):111-22. doi:10.1016/j. immuni.2007.05.016

48. Hammond MEW, Lapointe GR, Feucht PH, Hilt S, Gallegos CA, Gordon CA, et al. Il-8 Induces neutrophil chemotaxis predominantly via type-I Il-8 receptors. J Immunol (1995) 155(3):1428-33.

49. Van Damme J, Proost P, Lenaerts JP, Opdenakker G. Structural and functional identification of two human, tumor-derived monocyte chemotactic proteins (MCP-2 and MCP-3) belonging to the chemokine family. J Exp Med (1992) 176(1):59-65. doi:10.1084/jem.176.1.59

50. Tsai SF, Tao M, Ho LI, Chiou TW, Lin SZ, Su HL, et al. Isochaihulactoneinduced DDIT3 causes ER stress-PERK independent apoptosis in glioblastoma multiforme cells. Oncotarget (2016) 8(3):4051-61. doi:10.18632/ oncotarget.13266

51. Feder ME, Hofmann GE. Heat-shock proteins, molecular chaperones, and the stress response: evolutionary and ecological physiology. Annu Rev Physiol (1999) 61:243-82. doi:10.1146/annurev.physiol.61.1.243

52. Irmler $M$, Thome $M$, Hahne $M$, Schneider $P$, Hofmann K, Steiner V, et al. Inhibition of death receptor signals by cellular FLIP. Nature (1997) 388(6638):190-5. doi:10.1038/40657

53. Ma O, Cai WW, Zender L, Dayaram T, Shen J, Herron AJ, et al. MMP13, Birc2 (cIAP1), and Birc3 (cIAP2), amplified on chromosome 9, collaborate with p53 deficiency in mouse osteosarcoma progression. Cancer Res (2009) 69(6):2559-67. doi:10.1158/0008-5472.CAN-08-2929

54. Rahman N, Stewart G, Jones G. A role for the atopy-associated gene PHF11 in T-cell activation and viability. Immunol Cell Biol (2010) 88(8):817-24. doi:10.1038/icb.2010.57

55. Hashiguchi M, Kobori H, Ritprajak P, Kamimura Y, Kozono H, Azuma M. Triggering receptor expressed on myeloid cell-like transcript 2 (TLT-2) is a counter-receptor for B7-H3 and enhances T cell responses. Proc Natl Acad Sci U S A (2008) 105(30):10495-500. doi:10.1073/pnas.0802423105

56. Sanchez A, Kiley MP. Identification and analysis of Ebola virus messenger RNA. Virology (1987) 157(2):414-20. doi:10.1016/0042-6822(87)90283-2

57. Schornberg K, Matsuyama S, Kabsch K, Delos S, Bouton A, White J. Role of endosomal cathepsins in entry mediated by the Ebola virus glycoprotein. J Virol (2006) 80(8):4174-8. doi:10.1128/JVI.80.8.4174-4178.2006 
58. Wahl-Jensen V, Kurz S, Feldmann F, Buehler LK, Kindrachuk J, DeFilippis V, et al. Ebola virion attachment and entry into human macrophages profoundly effects early cellular gene expression. PLoS Negl Trop Dis (2011) 5(10):e1359. doi:10.1371/journal.pntd.0001359

59. Escudero-Perez B, Volchkova VA, Dolnik O, Lawrence P, Volchkov VE. Shed GP of Ebola virus triggers immune activation and increased vascular permeability. PLoS Pathog (2014) 10(11):e1004509. doi:10.1371/journal.ppat.1004509

60. Pulit-Penaloza JA, Scherbik SV, Brinton MA. Type 1 IFN-independent activation of a subset of interferon stimulated genes in West Nile virus Eg101-infected mouse cells. Virology (2012) 425(2):82-94. doi:10.1016/j. virol.2012.01.006

61. Spurgers KB, Alefantis T, Peyser BD, Ruthel GT, Bergeron AA, Costantino JA, et al. Identification of essential filovirion-associated host factors by serial proteomic analysis and RNAi screen. Mol Cell Proteomics (2010) 9(12): 2690-703. doi:10.1074/mcp.M110.003418

62. Reid SP, Shurtleff AC, Costantino JA, Tritsch SR, Retterer C, Spurgers KB, et al. HSPA5 is an essential host factor for Ebola virus infection. Antiviral Res (2014) 109:171-4. doi:10.1016/j.antiviral.2014.07.004

63. Cilloniz C, Ebihara H, Ni C, Neumann G, Korth MJ, Kelly SM, et al. Functional genomics reveals the induction of inflammatory response and metalloproteinase gene expression during lethal Ebola virus infection. J Virol (2011) 85(17):9060-8. doi:10.1128/JVI.00659-11

64. Ruibal P, Oestereich L, Ludtke A, Becker-Ziaja B, Wozniak DM, Kerber R, et al. Unique human immune signature of Ebola virus disease in Guinea. Nature (2016) 533(7601):100-4. doi:10.1038/nature17949

65. Liu X, Speranza E, Munoz-Fontela C, Haldenby S, Rickett NY, GarciaDorival I, et al. Transcriptomic signatures differentiate survival from fatal outcomes in humans infected with Ebola virus. Genome Biol (2017) 18(1):4. doi:10.1186/s13059-016-1137-3
66. Kash JC, Walters KA, Kindrachuk J, Baxter D, Scherler K, Janosko KB, et al. Longitudinal peripheral blood transcriptional analysis of a patient with severe Ebola virus disease. Sci Transl Med (2017) 9(385):eaai9321. doi:10.1126/ scitranslmed.aai9321

67. Hilkens CM, Schlaak JF, Kerr IM. Differential responses to IFN-alpha subtypes in human T cells and dendritic cells. J Immunol (2003) 171(10):5255-63. doi:10.4049/jimmunol.171.10.5255

68. Wilson EB, Yamada DH, Elsaesser H, Herskovitz J, Deng J, Cheng G, et al. Blockade of chronic type I interferon signaling to control persistent LCMV infection. Science (2013) 340(6129):202-7. doi:10.1126/science.1235208

69. Teijaro JR, Ng C, Lee AM, Sullivan BM, Sheehan KC, Welch M, et al. Persistent LCMV infection is controlled by blockade of type I interferon signaling. Science (2013) 340(6129):207-11. doi:10.1126/science.1235214

70. Leung LW, Park MS, Martinez O, Valmas C, Lopez CB, Basler CF. Ebolavirus VP35 suppresses IFN production from conventional but not plasmacytoid dendritic cells. Immunol Cell Biol (2011) 89(7):792-802. doi:10.1038/icb. 2010.169

Conflict of Interest Statement: The authors declare that the research was conducted in the absence of any commercial or financial relationships that could be construed as a potential conflict of interest.

Copyright (c) 2017 Menicucci, Versteeg, Woolsey, Mire, Geisbert, Cross, Agans, Jankeel, Geisbert and Messaoudi. This is an open-access article distributed under the terms of the Creative Commons Attribution License (CC BY). The use, distribution or reproduction in other forums is permitted, provided the original author(s) or licensor are credited and that the original publication in this journal is cited, in accordance with accepted academic practice. No use, distribution or reproduction is permitted which does not comply with these terms. 